\title{
Generation of a train of three-dimensional optical solitons in a self-focusing medium
}

\author{
Nail Akhmediev \\ Optical Sciences Centre, Institute of Advanced Studies, The Australian National University, \\ Canberra, Australian Capital Territory 2601, Australia \\ J. M. Soto-Crespo \\ Instituto de Optica, Consejo Superior de Investigaciones Científicas, Serrano 121, 28006 Madrid, Spain
}

(Received 27 August 1992)

\begin{abstract}
The problem of modulation instability of a self-focused beam in a homogeneous nonlinear medium with saturation and anomalous group-velocity dispersion is solved numerically. It is shown that the results of this instability is beam breakup into a periodic train of three-dimensional (3D) spatial solitary waves. It is also shown that other types of periodic initial conditions can produce a periodic train of 3D spatial solitary waves. Our numerical simulations show that 3D solitary waves are attractors (foci or limit cycles) in the Hilbert space of solutions of the 3D nonlinear Schrödinger equation. A field of another configuration can converge to them upon propagation and after the emission of a certain amount of radiation.
\end{abstract}

PACS number(s): 42.65.Jx, 52.35.Mw, 03.40.Kf, 03.65.Ge

\section{INTRODUCTION}

There is currently great interest in three-dimensional (3D) localized pulses or "optical bullets" [1-3] in selffocusing media with anomalous group-velocity dispersion. It was shown in Ref. [3] that these 3D self-focused pulses are robust in the sense that they remain as separate solitary formations even after collisions. This behavior resembles that of solitons in 1D integrable systems, so we can call them "3D solitons." In fact, this terminology has become common since it was introduced in plasma theory [4]. Nevertheless, to avoid controversy or confusion we shall call them in the rest of this paper " $3 \mathrm{D}$ optical solitary waves" or simply "optical bullets." The question arises as to whether it is possible to generate these 3D solitary waves using the process of modulation instability of self-trapped beams. This type of modulation instability of the lowest-order self-trapped beam was first considered by Zakharov and Rubenchik [5] and studied in more detail in Ref. [6]. The effect of modulation instability on a $1 \mathrm{D} \mathrm{cw}$ wave in a single-mode optical fiber has been predicted [7], theoretically investigated [8], and experimentally observed $[9,10]$.

The aim of this paper is to investigate the modulation instability of self-focused beams in bulk, dispersive, nonlinear media and the process of generation of a train of $3 \mathrm{D}$ solitary waves as a result of this instability. We restrict ourselves to the case of saturable nonlinearity in this work to avoid the problem of beam collapse. The interesting phenomenon that we observed in our numerical simulations is the irreversible convergence of the solution to the regular train of optical bullets. This convergence shows that, at least for some range of relevant parameters, optical bullets can serve as attractors. The fact that a soliton gas can be a statistical attractor in the asymptotic state of turbulence was shown in Ref. [11]. In con- trast to [11], (i) we start from regular initial conditions which give the regular behavior of solitons, and (ii) in our case the soliton part of the field becomes completely separated from the radiative part during propagation.

We write the nonlinear wave equation in a slowly varying field-amplitude approximation, taking into account the effects of diffraction and dispersion to second order. In the reference frame moving along the $z$ axis with the light group velocity, this equation takes the following form [6]:

$$
i \psi_{z}+\Delta_{r} \psi+\psi_{\tau \tau}-\psi+\frac{|\psi|^{2} \psi}{1+\beta|\psi|^{2}}=0
$$

where we take the transverse Laplacian $\Delta_{r} \psi=\psi_{r r}+(1 / r) \psi_{r}$ to be independent of the angular variable, $z$ is the normalized longitudinal coordinate, $\tau$ is the normalized retarded time, and $\beta$ is the saturation parameter. We assume, as in $[1,3]$, that the group-velocity dispersion is negative. The function $\psi$ and all variables in Eq. (1) are normalized to eliminate most of the parameters of the problem. Only the saturation parameter $\beta$ remains.

There exist solutions of Eq. (1) having the form of pulses localized in three dimensions. For any solution localized in three dimensions, Eq. (1) has the invariant

$$
I=2 \pi \int_{0}^{\infty} r d r \int_{-\infty}^{\infty} d \tau|\psi(r, \tau)|^{2},
$$

which does not depend on $z$. So, the system described by Eq. (1) is Hamiltonian. We shall call $I$ the "energy invariant."

Equation (1) also has stationary (independent of $z$ and $t$ ) solutions localized in two transverse dimensions [12]. These stationary solutions $\psi_{0}(r)$ can be found by solving Eq. (1), from which the terms with derivatives on $z$ and $t$ are excluded: 


$$
\Delta_{r} \psi_{0}-\psi_{0}+\frac{\left|\psi_{0}\right|^{2} \psi_{0}}{1+\beta\left|\psi_{0}\right|^{2}}=0
$$

In this paper we consider only the lowest-order nodeless solution of Eq. (3) with zero boundary conditions. For this stationary solution, $\psi_{0}(r)$, the quantity

$$
I=2 \pi T \int_{0}^{\infty}\left|\psi_{0}(r, \tau)\right|^{2} r d r
$$

represents the energy invariant of the beam per unit length $T$ along the $\tau$ axis. In the case of periodic solutions along the $\tau$ variable, $T$ is equal to the period.

\section{OPTICAL BULLETS}

Following Silberberg [1], we can give the name optical bullets to the solutions of Eq. (1) which are in the form of 3D solitary waves:

$$
\psi(r, \tau, z)=U(\rho) \exp [i(\alpha-1) z],
$$

where $\rho=\left(r^{2}+\tau^{2}\right)^{1 / 2}$ and $\alpha$ is the propagation constant. The reason for taking $(\alpha-1)$ in the exponent of Eq. (5) is that in deriving Eq. (1) the phase factor $\exp (i z)$ has already been used. Now Eq. (1) reduces to

$$
U_{\rho \rho}+\frac{2}{\rho} U_{\rho}-\alpha U+\frac{|U|^{2} U}{1+\beta|U|^{2}}=0
$$

Apart from a constant factor in front of the first and the second terms in Eq. (6) and the denominator of the nonlinear term, this equation coincides with Eq. (3) of Ref. [1].

The energy invariant $I$ for optical bullets in spherical coordinates takes the form

$$
I=4 \pi \int_{0}^{\infty}|U|^{2} \rho^{2} d \rho .
$$

The energy invariant versus propagation constant $\alpha$ for optical bullets described by Eq. (6) is shown in Fig. 1(a). Note that for any value of the energy invariant higher than some $I_{\min }$, there are two corresponding values of the propagation constant $\alpha$. We can expect [13] that the branch of this curve with positive slope (solid curve) is stable and that the branch with negative slope (dashed curve) is unstable. The meaning of the points $A$ and $B$ on this diagram will be explained later. The curve for half width at half maximum for these solutions versus its maximal amplitude $U_{0}=U(\rho=0)$ is shown in Fig. 1(b). This figure shows that an optical bullet becomes wider at low and at high intensities, having its smallest size at some medium intensity. This is the main difference of optical bullets in a saturable medium from those in a nonlinear medium with Kerr law. We should remark that the two minima in these two curves correspond to different solutions, that is, the point of minimum of the curve in Fig. 1(b) is not the point where the stability of the solution changes.

\section{MODULATION INSTABILITY OF SELF-FOCUSED BEAMS}

We are interested in solutions of Eq. (1) with initial condition (at $z \approx 0$ ) of the form

$$
\psi(r, \tau, z)=\psi_{0}(r)+\mu f(r) \cos (\gamma \tau) \exp (\delta z),
$$

where $\mu$ is a small parameter, $\gamma=2 \pi / T$ is the frequency of a periodic modulation, $\delta=\delta(\gamma)$ is the modulation growth rate, and $f(r)$ is the perturbation function. This function is complex in general. The functions $f(r)$ and $\delta(\gamma)$ have been calculated in Ref. [6]. As an illustrative example we show in Fig. 2 the stationary $\mathrm{cw}$ solution $\psi_{0}$ for $\beta=0.05$ and the corresponding perturbative function $f(r)$ for $\gamma=1.428(T=4.4)$. For this frequency, the perturbation growth rate $\delta(1.428)$ is a maximum and is equal to 2.172 . In this paper we restrict ourselves to the case of maximal growth rate.

We have solved Eq. (1) numerically using a split step method. We divide the equation into a dispersive part and a diffractive and nonlinear part. At each step we
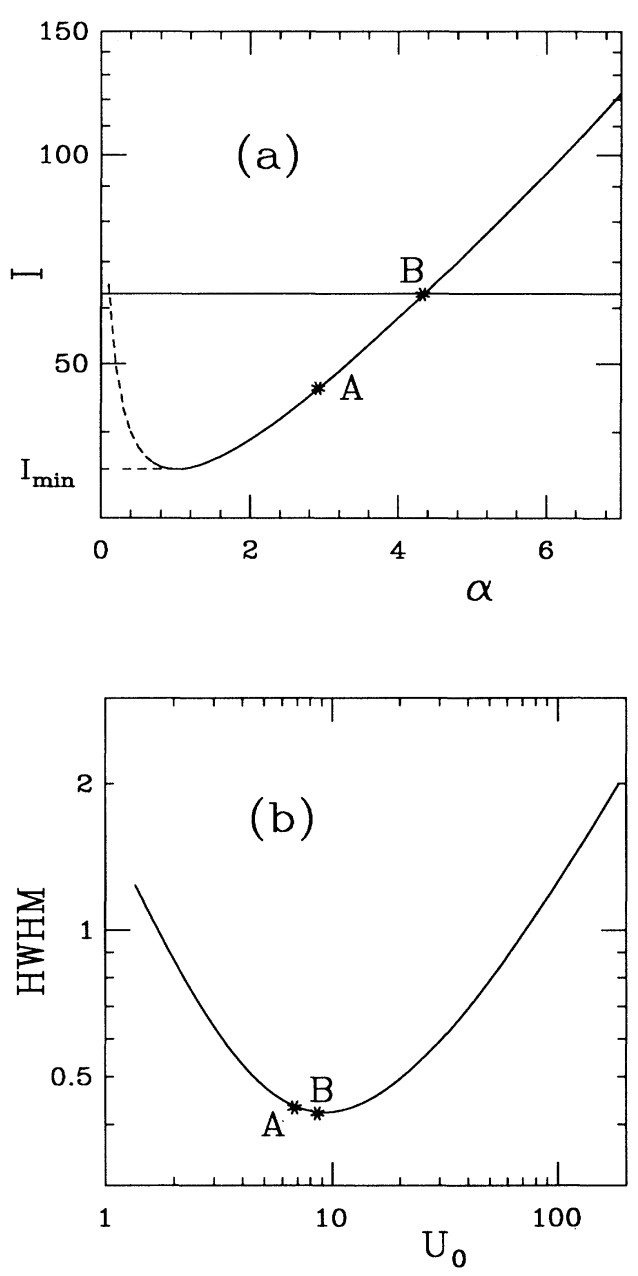

FIG. 1. (a) Energy vs propagation constant for optical bullets. The curve has stationary (with positive slope) as well as nonstationary (negative slope) branches. The horizontal line (and point $B$ ) in this figure correspond to the average initial energy contained in a period of modulation for the case studied in the text. Point $A$ corresponds to the energy contained in each optical bullet after break up. See text. (b) Half width at half maximum vs maximum amplitude of optical bullets. 


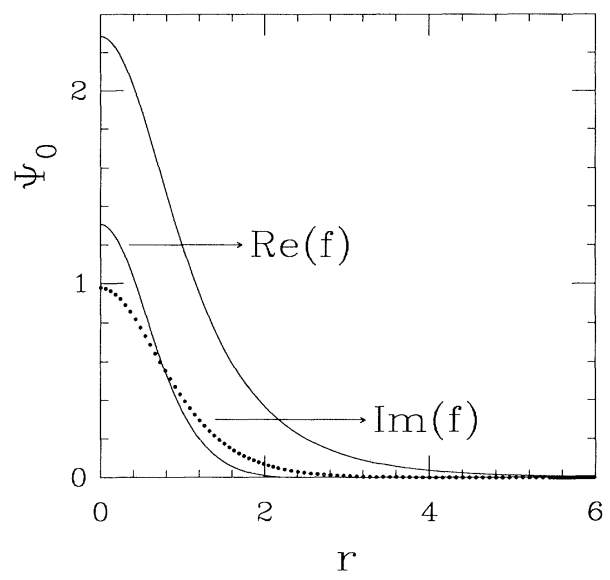

FIG. 2. Real (solid line) and imaginary (dotted line) parts of the perturbation along with the shape of the initial self-focused beam.

solve the dispersive part using the fast Fourier transform which automatically imposes time periodic boundary conditions. The diffractive plus the nonlinear part is solved using a Crank-Nicholson scheme with zero boundary condition at the maximum value of $r$ considered. The grid size was chosen to have up to 2000 points in the $r$ direction and up to 256 (usually 128 ) points in the $\tau$ direction. The step size in the $z$ direction was typically taken to be $\Delta z=0.01$. Numerical accuracy was checked by repeating the simulations for different grid sizes and step sizes. In most of the simulations we used zero boundary conditions at $r=80$. We used saturation parameter $\beta=0.05$ throughout most of the simulations. In what follows, we present our numerical results.

To ensure the reliability of our code we first considered initial conditions in the form of optical bullets:

$$
\psi(r, \tau, z=0)=U(\rho, \alpha)
$$

and we verified that they propagate long distances (up to $z=100$ ) without any visible change in both their temporal and spatial profiles. We used values of $\alpha$ for these simulations on the branch of energy dispersion curve [Fig. 1(a)] with positive slope, particularly $\alpha=2.94$ and 4.35 [points $A$ and $B$ in Fig. 1(a)]. These runs serve to confirm that optical bullets are stable on the positive slope branch of this curve. As a second verification of our program we also consider as an initial condition stationary $\mathrm{cw}$ solutions with time periodic perturbations of frequency higher than the limit frequency of instability, as perturbatively calculated in Ref. [6]. Again the numerical results agreed with the linear perturbative analysis and the modulation did not grow.

Figure 3 shows an example of the evolution of the field intensity $|\psi|^{2}$, corresponding to the initial condition given by Eq. (8), in the point of its maximum amplitude $(r=0, \tau=0)$. The modulation period was $T=4.4$ and $\psi_{0}(r)$ and $f(r)$ are those shown in Fig. 2. As one would expect, for very small parameters $\mu(=0.00001$ in this particular case) the field remains as a stationary solution

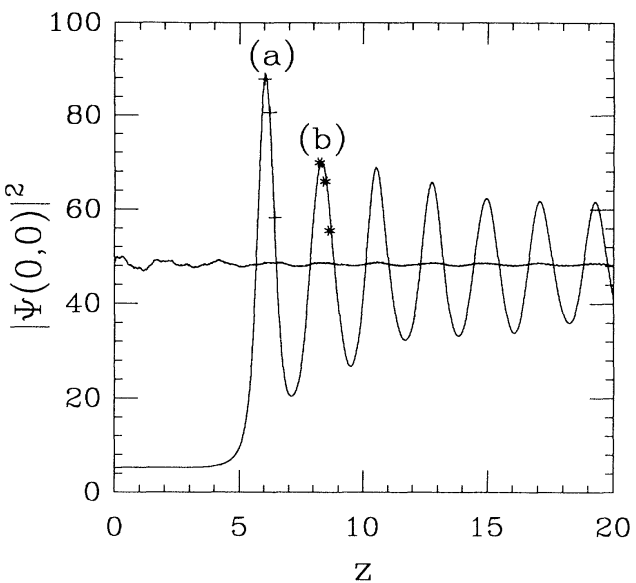

FIG. 3. Field intensity $|\psi|^{2}$ in the point $(\tau=0, r=0)$ vs the propagation distance $z$ for the initial condition given by the Eq. (8). The modulation period is $T=4.4$. The length of the initial part of this curve (with exponentially growing perturbation) depends on the initial amplitude of modulation $\mu$ and is approximately equal to 5 in this particular case $(\mu=0.00001)$. The roughly horizontal line corresponds to the peak intensity of the optical bullet, $|U(\rho=0)|^{2}$, with propagation constant $\alpha=2.94$.

for long distances (up to $z=5$ ), until the exponential factor in Eq. (8) becomes large enough. When the exponent in Eq. (8) becomes comparable with $\mu^{-1}$, the field intensity increases rapidly, reaching a maximum value [point (a) in Fig. 3], and then the behavior of the field intensity becomes oscillatory with an amplitude that decays smooth1y. For larger values of $\mu$ (we used the values up to $\mu=0.5$ ) the initial stationary part of the curve in Fig. 3 decreases in length, but the rest of the curve in Fig. 3 is hardly influenced by $\mu$. At point $(a)$, the field in each period is highly compressed in three dimensions and the whole solution looks like a train of three-dimensional self-compressed pulses. Beginning from point $(a)$, the degree of compression decreases up to the point of the minimum of the curve in Fig. 3, where pulses become more spread out but are still well separated. After the minimum point in Fig. 3, the pulses in each period are compressed again up to the point of the next maximum [point $(b)$ in Fig. 3]. The degree of compression is now less than that at point $(a)$. The process is repeated again in the next period, but with lower amplitude. One can see that the initially $\mathrm{cw}$ beam is now broken up into separate $3 \mathrm{D}$ optical solitary waves which oscillate on propagation.

The horizontal line in Fig. 3 (at intensity level $\approx 48.3$ ), around which oscillations take place, corresponds to the peak intensity $|U(\rho=0)|^{2}$ of the optical bullet having propagation constant $\alpha=2.94$ [point $A$ in Fig. 1(a)]. We can suppose that oscillations in Fig. 3 converge to this value. Field envelope oscillations also occur around a mean shape corresponding to this optical bullet. To see this, we present in Fig. 4 the intensity profile at $r=0$ in one period of $\tau$, for $z$ values corresponding to the point of minimum $(z=13.9)$ and maximum $(z=15)$ of the curve 


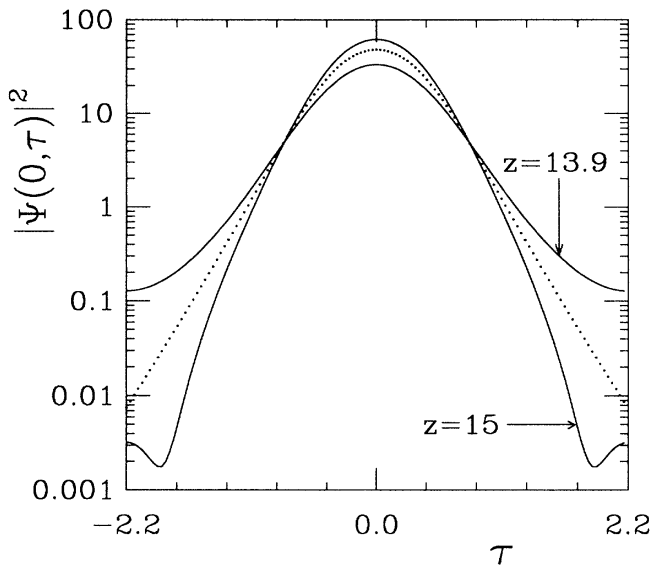

FIG. 4. Temporal shape at $r=0$ of the beam (solid curves) for two different values of the propagation distance $z$ (shown above the curves). The shape oscillates upon propagation around a mean value given by the profile of the optical bullet which has a propagation constant $\alpha=2.94$ (dotted curve).

in Fig. 3. Note the logarithmic scale in the vertical axis of this figure. The shape of the optical bullet (the dotted curve) is also shown for comparison. The intensity profile at the midpoint of the oscillations $(z \approx 15.45)$, between the maximum $(z \approx 15)$ and the next minimum, becomes spherically symmetric (in the chosen frame) and almost coincides with the shape of an optical bullet. This can be seen clearly in Fig. 5. This figure shows both the $r$ and $\tau$ intensity profiles (solid lines), along with the intensity profile for an optical bullet (dotted line) on the same plot. The intensity scale is linear in this plot. The three curves are indistinguishable. Of course the negative part of the $x$ axis is only for the $\tau$ variable.

At each oscillation, a small amount of energy is radiat-

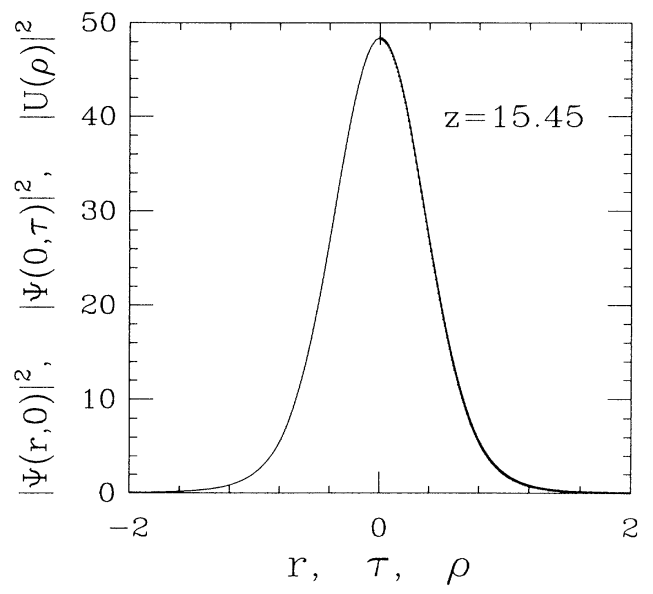

FIG. 5. Temporal and radial profiles of the 3D solitary waves generated after propagating a distance $z=15.45$ (solid curves). The dotted line represents the profile of the corresponding optical bullet for $\alpha=2.94$. ed from the beam. This process can be seen in Figs. 6(a) and $6(\mathrm{~b})$. The heavy solid curve in these figures is the curve for the initial stationary solution $\psi_{0}(r)$. The other curves represent the field intensity profiles for $\tau=0$ at three difference values of $z$ close to the first [Fig. 6(a)] and to the second [Fig. 6(b)] maximum of the curve in Fig. 3. The rings produced are propagating out of the central beam, increasing their radii and diffracting away. Other rings are emitted following each oscillation, and these interfere with each other. The radiation of many rings and the result of their interference can be seen clearly in Fig. 7 , where we have plotted the intensity profile at $z=14$ (the minimum point), 15 (the maximum point), and 14.5 (the intermediate point).

As a consequence of this radiation process, the energy in each period in $\tau$ decreases with each oscillation. The horizontal line in Fig. 1(a), at an energy level $I=62.9$, represents the energy per period corresponding to the initial stationary cw beam. Point $A$ in the same figure, at an energy level $I=46.3$, corresponds to the optical bullet to which the solution is converging. Hence approximately
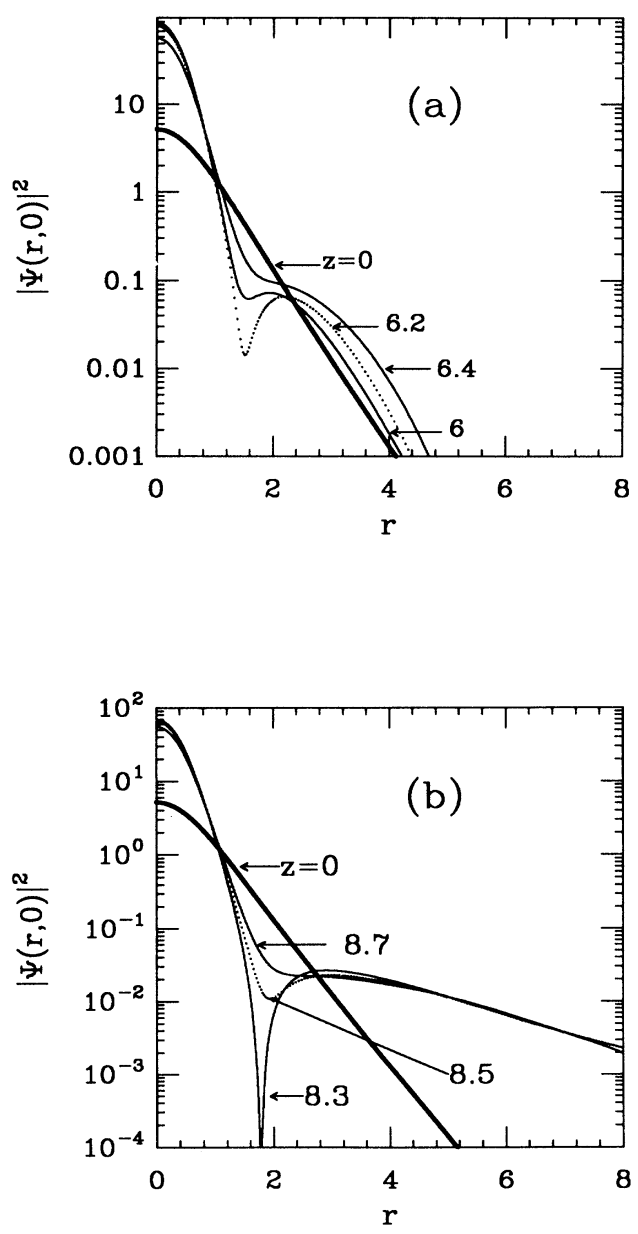

FIG. 6. Transverse (radial) profiles of the beam intensity at the propagation distances $z$ shown on each curve. They correspond to the (a) crosses and (b) asterisks of Fig. 3. 


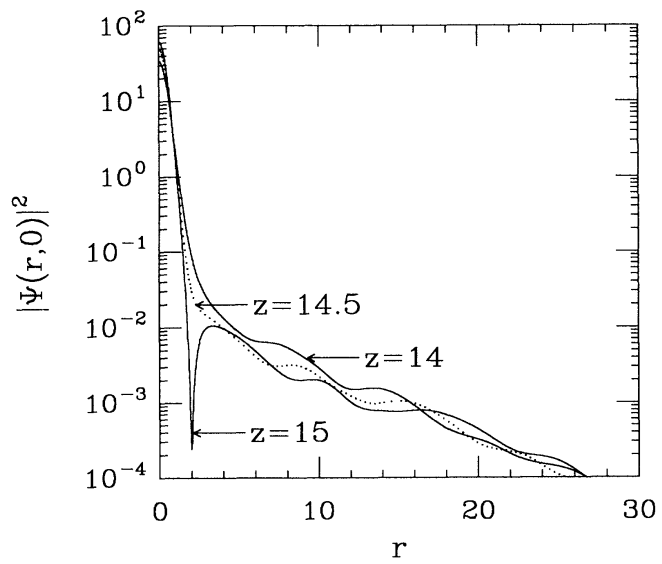

FIG. 7. The same as in Fig. 6 for $z=14,15$ (solid lines), and 14.5 (dotted line).

$26 \%$ of energy is radiated during the transition process from the $\mathrm{cw}$ beam to the train of optical bullets. It is interesting to note that the amount of radiation at each oscillation reduces almost to zero even if the oscillations are continuing with finite amplitude. Unfortunately we cannot investigate this peculiarity in more detail because the radiated field reaches the boundary and is reflected back after propagating a certain distance $z_{0}$.

\section{DISCUSSION}

We can see now that an initially homogeneous selffocused beam breaks up into separate light "clumps" as a result of the modulation instability, and that each of this clumps converges to a 3D solitary wave (optical bullet). Because of radiation, the process is apparently irreversible; this makes it different from the process of modulation instability in 1D [8]. There is no recurrence to the initial $\mathrm{cw}$ wave as occurs in the $1 \mathrm{D}$ case [8], nor is there a pseudorecurrence as in the case of transverse modulation instability in 2D [14]. An interesting situation now arises. Equation (1) that we are solving is Hamiltonian as it has an energy invariant (2). In a low-dimensional case, a trajectory starting from a saddle-type point usually arrives at the same or another saddle-type point with the same energy. In our case there is an important process of energy radiation, and then our solution loses energy. In the case of a bound initial conditions such as our beam, the radiation does not interact with the main beam after being emitted as it propagates away from it. A trajectory starting from a saddle-type point on one energy level cannot come back to any point on the same energy level. Instead of that, a trajectory can go to some special point on a different energy level. The final state could be a stationary solution or an oscillatory one close to an optical bullet. Therefore, in terms of nonlinear dynamical systems [15], our system evolves from a saddle-type point at a higher energy level to a focal point or to limit cycle on lower energy level.

To see this behavior of the solution in a more obvious way, we show in Fig. 8 the trajectory of the field $\psi$ at the point $(\tau=0, r=0)$ in the complex plane. The field, during

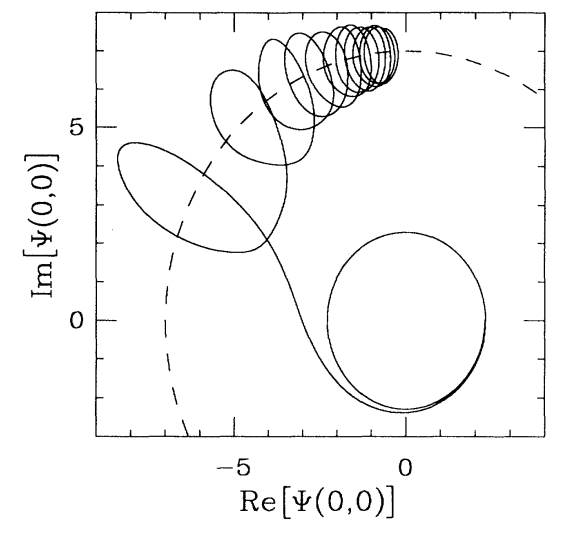

FIG. 8. Trajectory of the solution at the point $(r=0, \tau=0)$ on the complex plane. The data are presented in the frame with the additional phase factor $\exp (i 1.94 z)$. Trajectories for different points $r$ have the same qualitative behavior as that for $r=0$. The dotted line corresponds to the maximum amplitude of an optical bullet.

evolution accumulates phase, so the resulting field at the end of the process will have an additional rapidly oscillating factor, $\exp [i(\alpha-1) z]$, relative to the initial field. So the final limit cycle will be motionless in the complex plane which rotates rapidly relative to the initial plane where the saddle-type point is motionless. Physically, this means that during propagation, the propagation constant changes by certain amount $(\alpha-1)$. So we plot Fig. 8 in a rotating frame with phase factor $\exp (i 1.94 z)$. The corresponding optical bullet can be represented in this plot by any point at a fixed distance from the origin (a constant phase can be arbitrarily chosen). For a given $\alpha$, these points are located on the circle designated by a dotted line in Fig. 8. The trajectory of the field in our case converges to one of these points. So the optical bullets in a Hilbert space are the foci in the sense that they can attract the trajectories which go close enough to these points. Figure 8 shows an example of motion from one unstable stationary (saddle-type) point of Hilbert space to another stable focal point.

We also cannot neglect that limit cycles exist around these points. The solution can be periodic in nature, as happens in Fig. 8. In this case the final state is not the stationary solution, but the solution which oscillates around it with some small but finite perturbation. We cannot solve this problem in our numerical simulation because it requires quite long distances of propagation. In our numerical simulations this distance cannot exceed the value $z=50$ because reflections from the boundary $r=80$ could then influence the results. Anyway, our numerical simulations show that optical bullets (or oscillatory formations around them) are attractors in a Hilbert space of solutions of Eq. (1).

In order to show more clearly that envelope functions corresponding to optical bullets are the attractors in Hilbert space not only for the specific type of initial conditions [Eq. (8)] we used also the periodic initial condition having the following form: 


$$
\psi(r, \tau, z=0)=R(r) \cos \left(\frac{2 \pi}{T} \tau\right),
$$

where $R(r)$ is some radial envelope which contains enough energy to excite optical bullets in each half-period of the cosine function. Equation (9) describes optical beams with a double frequency carrier. This kind of periodic initial condition has been used for the generation of stable soliton trains in optical fibers [16]. In a 3D space, Eq. (10) is not a stationary solution. But for our purpose this is not important. If optical bullets can be excited with initial condition (10), then they could be excited using a wider spectra of initial conditions. For simplicity we used as an envelope the function $R(r)=\sqrt{2} \psi_{0}(r)$, where $\psi_{0}(r)$ is the stationary cw solution associated with $\beta=0.05$. We expect that consecutive optical bullets in the train (if they are created during propagation) would have their corresponding phases shifted by $\pi$. In order to have the same distance between optical bullets as in the previous cases, we have to choose $T=8.8$ in Eq. (10). This should provide the conditions for the formation of an optical bullet in each half-period of the function (10). The results of this numerical simulation are shown in Figs. 9 and 10.
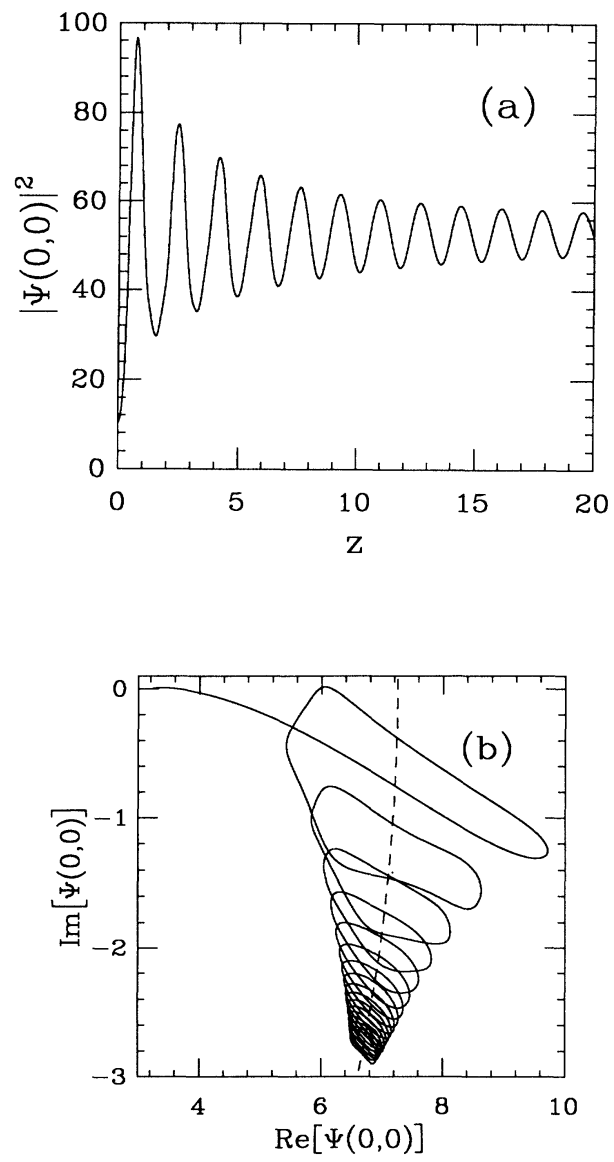

FIG. 9. (a) The same as in Fig. 3 and (b) the same as in Fig. 8 but for the initial condition given by Eq. (10).
The field intensity $|\psi|^{2}$ at the point $(r=0, \tau=0)$ versus the distance of propagation $z$, is shown in Fig. 9(a). The initial condition (10) is not close to any stationary solution, so it starts to change immediately at $z=0$. As in the previous case, the field oscillates around the average amplitude $|\psi|^{2} \approx 52$. The amplitude of these oscillations decreases in the same way as in the case shown in Fig. 3. The whole profile converges to the one corresponding to an optical bullet. The trajectory of this motion in complex space is shown in Fig. 9(b). It begins to oscillate around the focal point corresponding to an optical bullet almost immediately, without any transient like that shown in Fig. 8. The focal point, to which the trajectory is converging, rotates initially around the zero point, but fixes its position gradually in our rotating frame. The phase factor $\exp (i 2.17 z)$ is chosen to generate this graph, so that the final position of the focal point does not rotate in this frame. This indicates that the propagation constant of the optical bullets generated is equal to $\alpha=3.17$; this is different from the previous case. The corresponding point on the energy dispersion curve [Fig. 1(a)] is located slightly higher than point $A$. Although the resulting optical bullets for different initial conditions do not have to coincide, we can expect that the vicinity of point $\boldsymbol{A}$ is the most probable area of convergence of optical bullets originating from an arbitrary initial condition, if they carry enough energy to create the optical bullets. Figure 10 shows the field intensity distribution at $z=20$, which is the last point of the simulation presented in Fig. 9(a). The figure shows one period of the initial modulation. Two 3D solitary waves are located in each period as expected. The consecutive optical bullets have opposite phases, so that the trajectory of the second maximum $|\psi(0,4.4)|^{2}$, in the complex plane can be obtained by inverting the trajectory in Fig. 9(b) with respect to the origin.

Finally we should say that we have observed similar qualitative behavior for different values of the saturation parameter $\beta$. In particular for $\beta=0.2$, a much faster convergence towards an optical bullet appears and less energy $(10 \%)$ is radiated. On the other hand, for $\beta=0.5$ the oscillatory behavior of the initially stationary $\mathrm{cw}$ solution around an optical bullet was much more complicated and the convergence very poor.

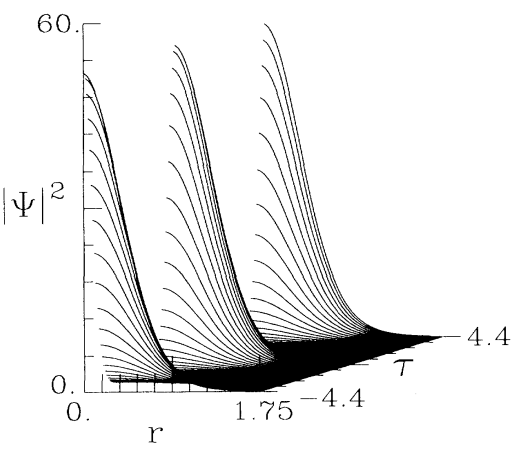

FIG. 10. Intensity distribution of the field in a period at $z=20$ for the same initial condition as in Fig. 9 . 


\section{CONCLUSIONS}

The train of separate optical bullets is produced due to the effect of modulation instability in the cases considered above. In some simulations we also used weakly modulated Gaussian beams. These beams also break up into a train of pulses with good convergence to optical bullets, if the initial Gaussian profile is close enough to the stationary solution. We thus expect that in a properly arranged experiment, using a medium with anomalous groupvelocity dispersion, any beam with a shape close to the self-focused one will break up into a train of optical bullets if it is slightly modulated in a certain range of frequencies.

In conclusion, we have solved numerically the problem of modulation instability of a self-focused beam in a homogeneous nonlinear medium with saturation. We have shown that the beam breaks up into a periodic train of 3D spatial solitary waves propagating with slowly decaying periodic oscillations. Other types of periodic initial conditions also can produce a periodic train of $3 \mathrm{D}$ spatial solitary waves. This shows that optical bullets are attractors in the Hilbert space of solutions of the 3D nonlinear Schrödinger equation; thus a range of other configurations can converge to them after propagation and the radiation of a certain amount of energy.

\section{ACKNOWLEDGMENTS}

J. M. S.-C. acknowledges a grant from the Ministerio de Educación y Ciencia of Spain.
[1] Y. Silberberg, Opt. Lett. 15, 1282 (1990).

[2] A. B. Blagoeva, S. G. Dinev, A. A. Dreischuh, and A. Naidenov, IEEE J. Quantum Electron. QE-27, 2060 (1991).

[3] D. E. Edmundson and R. H. Enns, Opt. Lett. 17, 588 (1992).

[4] V. E. Zakharov and E. A. Kuznetsov, Zh. Eksp. Teor. Phys. 66, 594 (1974) [Sov. Phys. —JETP 39, 285 (1974)].

[5] V. E. Zakharov and A. M. Rubenchik, Zh. Eksp. Teor. Phys. 65, 997 (1974) [Sov. Phys. -JETP 38, 494 (1973)].

[6] N. N. Akhmediev, V. I. Korneev, and R. F. Nabiev, Opt. Lett. 17, 393 (1992).

[7] A. Hasegawa and W. F. Brinkman, IEEE J. Quantum Electron. QE-16, 694 (1980).

[8] N. N. Akhmediev, V. M. Eleonskii, and N. E. Kulagin, Zh. Eksp. Teor. Fiz. 89, 1542 (1985) [Sov. Phys._JETP 62, 894 (1985)].

[9] K. Tai, A. Hasegawa, and A. Tomita, Phys. Rev. Lett. 56,
135 (1986).

[10] K. Tai, A. Tomita, J. L. Jewell, and A. Hasegawa, Appl. Phys. Lett. 49, 236 (1986).

[11] A. I. D'yachnko, V. E. Zakharov, A. N. Pushkarev, V. F. Shvets, and V. V. Yan'kov, Zh. Ekps. Teor. Fiz. 96, 2026 (1989) [Sov. Phys. —JETP 69, 1144 (1989)]; see also Pis'ma Zh. Eksp. Teor. Fiz. 48, 79 (1988) [JETP Lett. 48, 83 (1988)].

[12] R. Y. Chiao, E. Garmire, and C. H. Townes, Phys. Rev. Lett. 13, 479 (1964).

[13] A. A. Kolokolov, Zh. Prikl. Mekh. Tekh. Fiz. No. 3, 152 (1973) [Sov. J. Appl. Mech. Tech. Phys. No. 3, 426 (1973)].

[14] N. N. Akhmediev, D. R. Heatley, G. I. Stegeman, and E. M. Wright, Phys. Rev. Lett. 65, 1423 (1990).

[15] P. A. Cook, Nonlinear Dynamical Systems (Prentice-Hall International, Englewood Cliffs, NJ, 1986).

[16] P. V. Mamyshev, S. V. Chernikov, and E. M. Dianov, IEEE J. Quantum Electron. QE-27, 2347 (1991). 